\title{
Neural Network Control Based on Adaptive Observer for Quadrotor Helicopter
}

\author{
Hana Boudjedir ${ }^{1}$, Omar Bouhali ${ }^{1}$ and Nassim Rizoug ${ }^{2}$ \\ ${ }^{1}$ LAJ Lab, Automatic department, Jijel University, Algeria \\ hana_boudjedir@yahoo.fr, bouhali_omar@yahoo.fr \\ ${ }^{2}$ Mecatronic Lab, ESTACA School, Laval, France \\ nrizoug@yahoo.fr
}

\begin{abstract}
A neural network control scheme with an adaptive observer is proposed in this paper to Quadrotor helicopter stabilization. The unknown part in Quadrotor dynamical model was estimated on line by a Single Hidden Layer network. To solve the non measurable states problem a new adaptive observer was proposed. The main purpose here is to reduce the measurement noise amplification caused by conventional high gain observer by introducing some changes in observer's original structure that can minimize the variance and the amplitude of the noisy signal without increasing tracking error. The stability analysis of the overall closed-loop system/ observer is performed using the Lyapunov direct method. Simulation results are given to highlight the performances of the proposed scheme.
\end{abstract}

\section{KEYWORDS}

Adaptive observer; high gain observer; Neural Network control; Quadrotor; Lyapunov stability.

\section{INTRODUCTION}

As their application potential both in the military and industrial sector strongly increases, miniature unmanned aerial vehicles (UAV) constantly gain in interest among the research community [1]. Quadrotor Helicopter is considered as one of the most popular UAV platform. The main reasons for all this attention have stemmed from its simple construction and its large payload as compared with the conventional helicopter [2].

The Quadrotor is an under actuated system from where it has six degrees of freedom controlled only by four control inputs. To solve the Quadrotor UAV tracking control problem many techniques have been proposed [2-7] where the control objective is to control three desired Cartesian positions and a desired yaw angle.

In [1-3] Backstepping and sliding mode techniques have been used to control the Quadrotor. In [4] the $\mathrm{H} \infty$ robust control law was proposed and a PID and LQR controls have been applied in [5]. Unfortunately these techniques are limited if the dynamic model is completely or partially unknown and/or if the states variables are unavailable. 
The unknown nonlinear functions problem in the control law can be solved by using adaptive control technique based on universals approximators as artificial neural network [6]. Several Neural Networks adaptive schemes have been proposed for Quadrotor control [6-10].

To avoid the state variables measurement assumptions, state observers can be introduced in the control scheme such as in [9] where a neuro-sliding mode observer was proposed in order to reduce the noise measurement amplification. In [10] other observer was used to estimate the velocity, the corrective term here is the superposition of a observation errors proportional term and a feed-forward neural networks output used to estimate unknown functions.

High-gain observers have evolved over the past two decades as an important tool for the design of output feedback control of nonlinear systems [11]. However this observer has two drawbacks, the first one is the peak phenomena and the second one is its high sensitivity to noise measurements. Several methods have been proposed in the literature to reduce the last phenomena as in [11] were two observation gains values have been used. The biggest value was used in the transient response and the smallest observation gain was used in permanent regime. In [12] and [13] an adaptation laws is utilized to compute the optimal observation gain. However those methods present some limitations in particular when the observation gain has to be too large as in the case of quadrotor dynamic.

In the present paper a new robust adaptive scheme that does not require any prior information on model dynamics and state measurement is proposed. The SHL NN in the control loop is used to estimate the unknown part in Quadrotor's dynamic model. States variables reproduction is made by a proposed adaptive observer. The main purpose of the new observer is to reduce noise measurements amplification by the minimization of the variance and the amplitude of the noisy signal without introducing an increase in the tracking error. The learning law is derived based on the Lyapunov stability theory.

This paper is organized as follows. In Section 2, the dynamical model of the Quadrotor will be presented. Problem formulation will be posed in section 3. In Sections 4 and 5, neural adaptive controller based on high gain observer and the proposed observer will be developed respectively. Simulation results are given in section 6 to show the effectiveness and feasibility of the proposed observer at noise measurement presence. The conclusion is given in section 7 .

\section{QUADROTOR DYNAMICS}

Such its name indicates Quadrotor helicopter is composed of four propellers in cross configuration where each rotors pair situated on the same branch; turn in opposite direction of the other pair to avoid the device rotation on her (Figure. 1). 


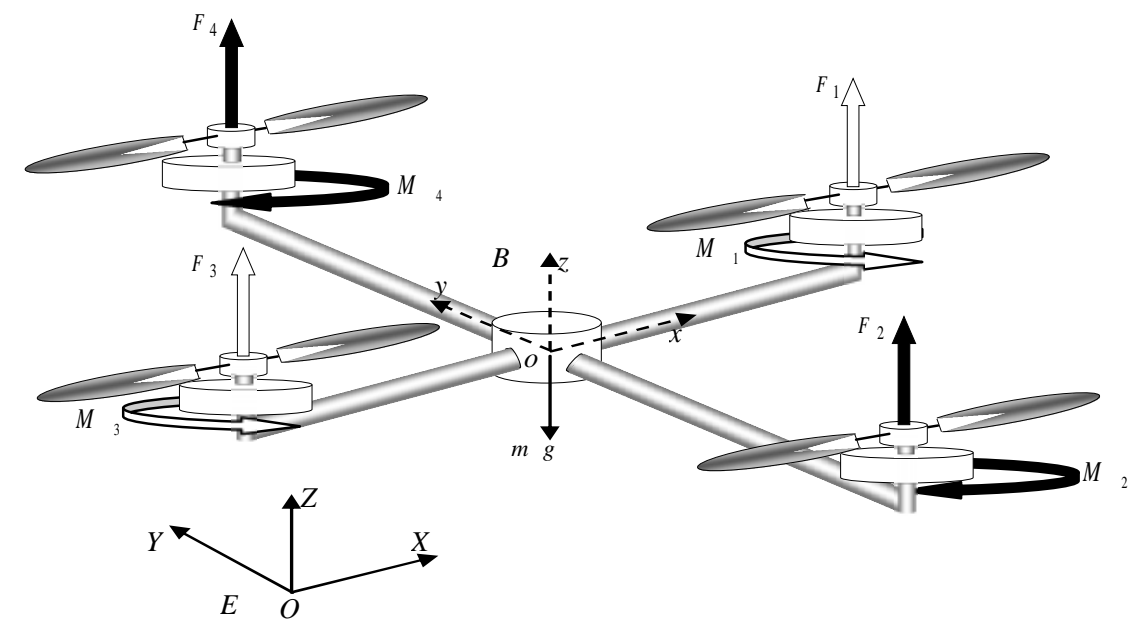

Figure 1. Model of conventional Quadrotor.

Flights mode in quadrotor are defined according to the direction and the velocity of each rotor. Vertical ascending (descending) flight is created by increasing (decreasing) thrust forces. Applying speed difference between front and rear rotors we will have pitching motion which is defined as a rotation motion around $Y$ axis coupled with a translation motion along $X$ axis. The same analogy is applied to obtain rolling motion, but by changing the side motors speed this time and as result we will have a rotation motion around $X$ axis coupled with a translation motion along $Y$ axis. The last flight mode is yaw motion; this one is obtained while increasing (decreasing) speed of motors (1.3) compared to (2.4) motors speed. Unlike pitch and roll motions, yaw rotation is the result of reactive torques produced by rotors rotation.

By using the formalism of Newton-Euler [2, 3, 6, 9] the dynamic model of a Quadrotor can be expressed as:

$$
\left\{\begin{array}{l}
\ddot{\varphi}=\frac{U_{\varphi}(t)}{I_{x}}+\frac{\left(I_{y}-I_{z}\right)}{I_{x}} \dot{\psi} \dot{\theta}-\frac{J_{r} \Omega_{r}(t)}{I_{x}} \dot{\theta}-\frac{k_{f r x}}{I_{x}} \dot{\varphi}^{2} \\
\ddot{\theta}=\frac{U_{\theta}(t)}{I_{y}}+\frac{\left(I_{z}-I_{x}\right)}{I_{y}} \dot{\psi} \dot{\varphi}+\frac{J_{r} \Omega_{r}(t)}{I_{y}} \dot{\varphi}-\frac{k_{f r y}}{I_{y}} \dot{\theta}^{2} \\
\ddot{\psi}=\frac{U_{\psi}(t)}{I_{z}}+\frac{\left(I_{x}-I_{y}\right)}{I_{z}} \dot{\varphi} \dot{\theta}-\frac{k_{f r z}}{I_{z}} \dot{\psi}^{2} \\
\ddot{x}=\frac{1}{m}\left(\left(C_{\psi} S_{\theta} C_{\varphi}+S_{\varphi} S_{\psi}\right) U_{1}(t)-k_{f f x} \dot{x}\right) \\
\ddot{y}=\frac{1}{m}\left(\left(S_{\psi} S_{\theta} C_{\varphi}-S_{\varphi} C_{\psi}\right) U_{1}(t)-k_{f t y} \dot{y}\right) \\
\ddot{z}=\frac{1}{m}\left(\left(C_{\theta} C_{\varphi}\right) U_{1}(t)-k_{f t z} \dot{z}\right)-g
\end{array}\right.
$$

Where : $m$ : Quadrotor mass, $k_{p}:$ Thrust factor, $k_{d}:$ drag factor, $\omega_{i}$ : angular rotor speed, $J=\operatorname{diag}\left(I_{x}\right.$, $\left.I_{y}, I_{z}\right)$ : inertia matrix, $K_{f t}=\operatorname{diag}\left(k_{f x x}, k_{f t y}, k_{f t z}\right)$, drag translation matrix, $K_{f r}=\operatorname{diag}\left(k_{f r x}, k_{f r y}, k_{f r z}\right)$ : friction aerodynamic coefficients, $\xi=\left[\begin{array}{lll}x & y & z\end{array}\right]^{T}$ : position vector, $\eta=\left[\begin{array}{lll}\varphi & \theta & \psi\end{array}\right]^{T}$ represents the angles of roll, pitch and yaw and $\Omega_{r}=\sum_{i=1}^{4}(-1)^{i+1} \omega_{i}$. 
The control inputs according to the angular velocities of the four rotors are given by:

$$
\left[\begin{array}{l}
U_{1}(t) \\
U_{\varphi}(t) \\
U_{\theta}(t) \\
U_{\psi}(t)
\end{array}\right]=\left[\begin{array}{cccc}
k_{p} & k_{p} & k_{p} & k_{p} \\
0 & -l k_{p} & 0 & l k_{p} \\
-l k_{p} & 0 & l k_{p} & 0 \\
k_{d} & -k_{d} & k_{d} & -k_{d}
\end{array}\right]\left[\begin{array}{c}
\omega_{1}^{2} \\
\omega_{2}^{2} \\
\omega_{3}^{2} \\
\omega_{4}^{2}
\end{array}\right]
$$

\subsection{Virtual control}

In this section, three virtual control inputs will be defined to ensure that the Quadrotor follows a specified trajectory. Those virtual controls are [9]:

$$
\left\{\begin{array}{l}
U_{x}(t)=\left(C_{\psi} S_{\theta} C_{\varphi}+S_{\varphi} S_{\psi}\right) U_{1}(t) \\
U_{y}(t)=\left(S_{\psi} S_{\theta} C_{\varphi}-S_{\varphi} C_{\psi}\right) U_{1}(t) \\
U_{z}(t)=\left(C_{\theta} C_{\varphi}\right) U_{1}(t)
\end{array}\right.
$$

The physical interpretation of theses virtual control, means that the control of translation motion depends on three common inputs are: $\theta, \varphi$ and $U_{l}(t)$. This requires that the rolling and pitching motions must take a desired trajectory to guarantee the control task of translation motion. Using equation (3) the desired trajectories in rolling and pitching are defined as follow [9]:

$$
\left\{\begin{array}{l}
\varphi_{d}=\arcsin \left(\frac{U_{x}(t) S_{\psi d}-U_{y}(t) C_{\psi d}}{\sqrt{U_{x}^{2}(t)+U_{y}^{2}(t)+U_{z}^{2}(t)}}\right) \\
\theta_{d}=\arctan \left(\frac{C_{\psi d} U_{x}(t)+S_{\psi d} U_{y}(t)}{U_{z}(t)}\right)
\end{array}\right.
$$

with $\varphi_{d}, \theta_{d}$ and $\psi_{d}$ are the desired trajectories in roll, pitch and yaw respectively.

\section{Problem Formulation}

After the use of the virtual control defined in eq. (3), the Quadrotor helicopter dynamical model can be described by the following differential equation system:

$$
\left\{\begin{array}{l}
y_{1}^{\left(r_{1}\right)}=f_{1}(X)+g_{1}(X) u_{1}(t) \\
\vdots \\
y_{p}^{\left(r_{p}\right)}=f_{p}(X)+g_{p}(X) u_{p}(t)
\end{array}\right.
$$

Where the state space form is represented by:

$$
\Sigma_{i}=\left\{\begin{array}{l}
\dot{x}_{i 1}=x_{i 2} \\
\vdots \\
\dot{x}_{i r_{i}}=f_{i}(X)+g_{i}(X) u_{i}(t) \\
y_{i}=x_{i, 1}+b_{i}
\end{array}\right.
$$


International Journal of Information Technology, Control and Automation (IJITCA) Vol.2, No.3, July 2012

with: $X=\left[\begin{array}{lllllll}y_{1} & \dot{y}_{1} & \cdots & y_{1}^{\left(r_{1}-1\right)}, \cdots, y_{p} & \dot{y}_{p} & \cdots & y_{p}^{\left(r_{p}-1\right)}\end{array}\right]^{T}$ : state space vector, $U=\left[\begin{array}{lll}u_{1} & \cdots & u_{p}\end{array}\right]^{T}:$ the input vector, $Y=\left[\begin{array}{lll}y_{1} & \cdots & y_{p}\end{array}\right]^{T}$ : output vector which is assumed available for measurement, $f_{i}(X)$, $g_{i}(X)$ are smooth nonlinear unknown functions and $b_{i}$ is the measurement noise.

Assumption.1: The desired output trajectory $y_{d i}, i=\overline{1: p}$ and its first $r_{i}$ derivatives are smooth and bounded.

Assumption.2: The gain $g_{i}(X)$ is bounded, positive definite and slowly time varying.

Tracking error $e_{i}$ and filtered errors $s_{i}$ are defined by following equation:

$$
\left\{\begin{array}{l}
e_{i}=y_{d i}(t)-y_{i}(t) \\
s_{i}=\left(\frac{d}{d t}+\lambda_{i}\right)^{r_{i}-1} e_{i}=\Phi_{\mathrm{i}} E_{i}, \quad \lambda_{i}>0
\end{array}\right.
$$

From filtered error expression we can conclude that the convergence of tracking error and its derivatives to zero is guaranteed when $s_{i}$ converge to zero [14]. For that raison, our control objective will be based on the synthesis of control law that allows the convergence to zero of the filtered error.

The filtered errors time derivative of can be expressed by:

$$
\dot{s}_{i}=v_{i}-f_{i}(X)-g_{i}(X) u_{i}(t)=e_{i}^{\left(r_{i}\right)}+\Phi_{0 i} E_{i}, \quad i=\overline{1: p}
$$

With:

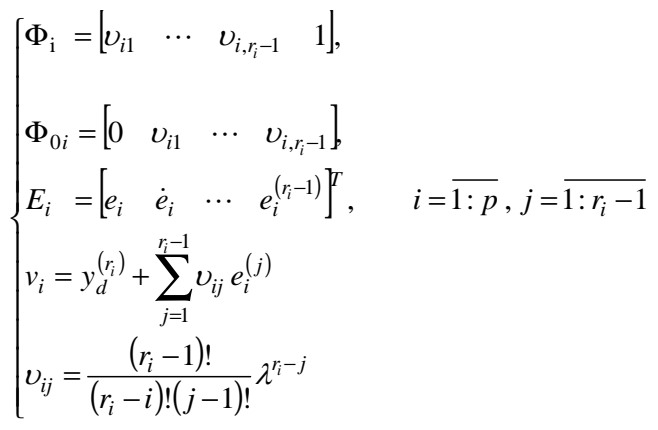

The following equation expresses the ideal control that can guarantee the closed loop system performances:

$$
u_{i}^{*}(t)=u_{i}^{e q^{*}}(t)+u_{i}^{p d^{*}}(t), i=\overline{1: p}
$$

With:

$$
\left\{\begin{array}{l}
u_{i}^{e q^{*}}(t)=g_{i}^{-1}(X)\left(v_{i}-f_{i}(X)\right) \\
u_{i}^{p d^{*}}(t)=k_{i} s_{i}=k_{i} \Phi_{i} E_{i} \quad, k_{i}>0
\end{array}\right.
$$

By substituting (10) in (8), we will have: 
$\dot{s}_{i}=-g_{i} k_{i} s_{i} \Rightarrow s_{i} \dot{s}_{i} \leq 0 \quad i=\overline{1: p}$

Which implying that $s_{i} \rightarrow 0$ when $t \rightarrow \infty$, and therefore $e_{i}^{(j)} \rightarrow 0 \quad j=\overline{1: r_{i}-1}, i=\overline{1: p}$ when $t \rightarrow \infty[14]$.

Unfortunately the application of this control law is impossible if the dynamic model and the state variables are unknown as this case. From this fact, six SHL NN will be implemented in control loop in order to approximate online the equivalent controller defined in (11). The non measured states problem will be solved by using a proposed observer.

\section{Synthesis of Neural Adaptive Control with a High Gain OBSERVER}

The proposed adaptive control is given by the following expression:

$$
\left\{\begin{array}{l}
u_{i}(t)=u_{i}^{e q}(t)+u_{i}^{r}(t)+u_{i}^{p d}(t) \\
u_{i}^{p d}(t)=k_{i} \hat{s}_{i}=k_{i} \Phi_{i} \hat{E}_{i} \\
u_{i}^{r}(t)=\hat{w}_{i} \operatorname{sign}\left(\hat{s}_{i}\right)
\end{array} \quad i=\overline{1: p}\right.
$$

Where:

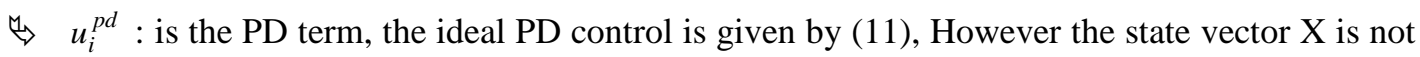
available to measure so $u_{i}^{p d^{*}}$ is replaced by $u_{i}^{p d}$,

$\Leftrightarrow u_{i}^{r}:$ is the robust term used for errors approximation compensation,

$\Leftrightarrow u_{i}^{e q}:$ is the neural adaptive control term which is an approximate of ideal equivalent control defined in (11).

$\Leftrightarrow$

The vector $\hat{E}_{i}$ is the estimated of $E_{i}$ defined in eq. (9) and $\hat{w}_{i} \in \mathfrak{R}$ is the estimated of $\bar{w}_{i}$, which will be defined later.

\section{1. Adaptive control Design}

According to approximation theorem [15] a SHL network presented in Figure.2 can approximate the unknown implicit ideal equivalent controller defined in (11) as follows:

$$
u_{i}^{e q^{*}}(t)=W_{i}^{T} \sigma\left(V_{i}^{T} \chi_{i}\right)+\varepsilon_{i} \quad i=\overline{1: p}
$$




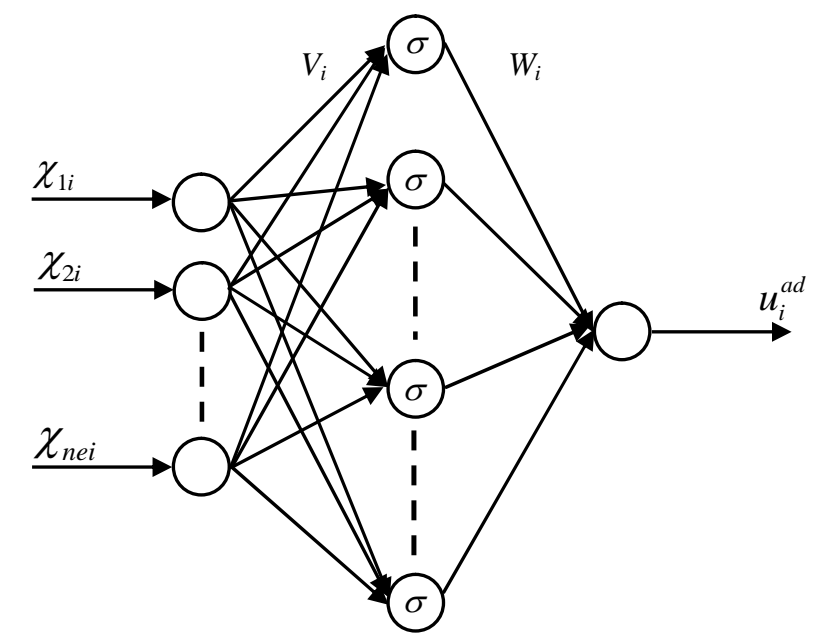

Figure 2: Network used in the control schemes.

where : $n e_{i}$ input neurons number, $n c_{i}$ hidden neurons number, $n s_{i}=1$ output neurons number, $\chi_{i}=\left[\begin{array}{lllll}\ddot{y}_{d i} & \dot{y}_{d i} & y_{d i} & e_{i} & \dot{e}_{i}\end{array}\right]^{T}$ input vector and $\sigma()$ is sigmoid activation function, $W_{i} \in \Re^{n c_{i} \times n s_{i}}$ and $V_{i} \in \Re^{n e_{i} \times n c_{i}}$ are the ideal weight and $\varepsilon_{i}$ is the reconstruction error.

Since the optimal weights and the input vector are unknown, it is necessary to estimate them by an adaptation mechanism so that the output feedback control law can be realized. $\hat{W}_{i}$ and $\hat{V}_{i}$ are the estimate of $W_{i}$ and $V_{i}$. Thus, the adaptive control approximating the ideal SHLNN output defined in (14) is given by :

$u_{i}^{e q}=\hat{W}_{i}^{T} \sigma\left(\hat{V}_{i}^{T} \hat{\chi}\right) \quad i=\overline{1: p}$

with : $\hat{\chi}_{i}=\left[\begin{array}{lllll}\ddot{y}_{d i} & \dot{y}_{d i} & y_{d i} & \hat{e}_{i} & \dot{\hat{e}}_{i}\end{array}\right]^{T}$ is $\chi_{i}$ estimated.

Assumption.3: $\left\|W_{i}\right\|_{F} \leq W_{i, m},\left\|V_{i}\right\|_{F} \leq V_{i, m}$. with : $W_{i, m}$ et $V_{i, m}$ are unknown positive constants.

The identification error of the equivalent control is :

$$
\begin{aligned}
\tilde{u}_{i}^{e q}(t) & =u_{i}^{e q}(t)-u_{i}^{e q}(t) \\
= & \tilde{W}_{i}^{T} \sigma\left(\hat{V}_{i}^{T} \hat{\chi}_{i}\right)+\hat{W}_{i}^{T} \sigma^{\prime}\left(\hat{V}_{i}^{T} \hat{\chi}\right) \tilde{V}_{i}^{T} \hat{\chi}_{i}+w_{i}
\end{aligned}
$$

Where, $w_{i}$ presents the estimation errors:

$$
w_{i}=\left(\begin{array}{l}
W_{i}^{T}\left\{\sigma\left(V_{i}^{T} \chi\right)-\sigma\left(V_{i}^{T} \hat{\chi}_{i}\right)\right\}+W_{i}^{T} O\left(\tilde{V}_{i}^{T} \hat{\chi}_{i}\right)^{2} \\
+\tilde{W}_{i}^{T} \sigma^{\prime}\left(\hat{V}_{i}^{T} \hat{\chi}_{i}\right) \tilde{V}_{i}^{T} \hat{\chi}_{i}+\varepsilon_{i}
\end{array}\right)
$$

Where: $\tilde{W}_{i}=W_{i}-\hat{W}_{i}$ and $\tilde{V}_{i}=V_{i}-\hat{V}_{i}$ are parameter estimation errors.

Assumption.4 : $\left\|w_{i}\right\| \leq \bar{w}_{i}$. with : $\bar{w}_{i}$ is an unknown positive constant.

To achieve the goal of controlling the weights adaptation laws are defined by: 


$$
\left\{\begin{array}{l}
\dot{\hat{W}}_{i}=F_{W i}\left(\sigma\left(\hat{V}_{i}^{T} \hat{\chi}_{i}\right) \hat{s}_{i}-\kappa_{i} \hat{W}_{i}\right) \\
\dot{\hat{V}}_{i}=F_{V i}\left(\hat{\chi}_{i} \hat{s}_{i} \hat{W}_{i}^{T} \sigma^{\prime}\left(\hat{V}_{i}^{T} \hat{\chi}_{i}\right)-\kappa_{i} \hat{V}_{i}\right)
\end{array}\right.
$$

with : $F_{W i}>0, F_{V i}>0$ are the adaptive gains and $\kappa_{i}$ is a positive constant. The estimated of $\bar{w}_{i}$ is given by:

$$
\dot{\hat{w}}_{i}=\gamma_{i}\left|\hat{s}_{i}\right| \quad, \gamma_{i}>0
$$

\section{2. High gain Observer Design}

The first time derivative of $E_{i}$ is giving by :

$$
\left\{\begin{array}{l}
\dot{E}_{i}=\left(A_{i}-B_{i} \Phi_{0 i}\right) E_{i}+B_{i} \dot{s}_{i} \\
e_{i}=C_{i} E_{i}
\end{array}\right.
$$

With:

$$
A_{i}=\left[\begin{array}{ccccc}
0 & 1 & 0 & \cdots & 0 \\
0 & 0 & 1 & \cdots & 0 \\
\vdots & \vdots & \vdots & \ddots & \vdots \\
0 & 0 & 0 & \cdots & 1 \\
0 & 0 & 0 & \cdots & 0
\end{array}\right], \quad B_{i}=\left[\begin{array}{c}
0 \\
0 \\
\vdots \\
0 \\
1
\end{array}\right], \quad C_{i}^{T}=\left[\begin{array}{c}
1 \\
0 \\
\vdots \\
0 \\
0
\end{array}\right]
$$

For this equation system; the conventional high gain observer used has the following form:

$$
\left\{\begin{array}{l}
\dot{\hat{E}}_{i}=\left(A_{i}-B_{i} \Phi_{0 i}\right) \hat{E}_{i}+\delta_{i} K_{i} \tilde{e}_{i} \\
\hat{e}_{i}=C_{i} \hat{E}_{i}
\end{array}\right.
$$

With:

$$
K_{i}=\left[\begin{array}{llll}
\vartheta_{i, 1} & \vartheta_{i, 2} \delta_{i} & \cdots & \vartheta_{i, r_{i}-1} \delta_{i}^{r_{i}-1}
\end{array}\right]^{T}
$$

And : $\delta_{i}>>1$ and $\vartheta_{i, 1}, \vartheta_{i, 2}, \cdots, \vartheta_{i, r_{i}-1}>0$ are design parameter. The notation $\hat{E}_{i}$ is the estimate of $E_{i}$, and $\tilde{e}_{i}=e_{i}-\hat{e}_{i}$.

As we already noted in first section this observer is characterized by its sensibility to noise measurement. For that raison a new adaptive observer is proposed in this paper.

\section{Proposed ObServer Design}

The proposed adaptive observer is given by the following equation: 
International Journal of Information Technology, Control and Automation (IJITCA) Vol.2, No.3, July 2012

$$
\left\{\begin{array}{l}
\dot{\hat{E}}_{i}=\left(A_{i}-B_{i} \Phi_{0 i}\right) \hat{E}_{i}+\mu_{i}(t) K_{i} \tilde{e}_{i}-\beta_{i} \mu_{i}(t) K_{i} e_{i} \\
\hat{e}_{i}=C_{i} \hat{E}_{i}
\end{array}\right.
$$

Where:

$$
\dot{\mu}_{i}=\varsigma_{i, 1}\left(\left|\tilde{e}_{i}\right|-\bar{\varepsilon}_{i}\right) \mu_{i}+\varsigma_{i, 2}\left(\mu_{i, f}-\mu_{i}\right)
$$

With: $\varsigma_{1, i}, \varsigma_{2, i}, \bar{\varepsilon}_{i}>0, \mu_{i}(0)=\delta_{i}, 1<\mu_{i, f}<\delta_{i}$, and, $\beta_{i} \in[0 ; 1[$.

In this case the filtered error dynamic can express by the following equation:

$$
\dot{s}_{i}=-g_{i}\left(k_{i} s_{i}-k_{i} \tilde{s}_{i}-w_{i, 1}\right)
$$

Where :

$$
w_{i, 1}=\left(\begin{array}{l}
\tilde{W}_{i}^{T} \sigma\left(\hat{V}_{i}^{T} \hat{\chi}_{i}\right)+\hat{W}_{i}^{T} \sigma^{\prime}\left(\hat{V}_{i}^{T} \hat{\chi}_{i}\right) \tilde{V}_{i}^{T} \hat{\chi}_{i} \\
+w_{i}-u_{i}^{r}
\end{array}\right)
$$

Assumption.5: $\left\|w_{i, 1}\right\| \leq c_{i, 1}\left\|\tilde{W}_{i}\right\|+c_{i, 2}\left\|\tilde{V}_{i}\right\|+c_{i, 3}$. With $: c_{i, 1}, \quad c_{i, 2}, \quad c_{i, 3}$ are unknown positive constants.

Assumption.6 : $\left|e_{i}\right| \leq \gamma_{i, 1}\left|s_{i}\right|+\gamma_{i, 2}$, with : $\gamma_{i, 1}, \gamma_{i, 2}>0$.

The observer error dynamic is given by :

$$
\dot{\tilde{E}}_{i}=\mu_{i}(t) \bar{A}_{i} \tilde{E}_{i}-B_{i} \Phi_{0 i} \tilde{E}_{i}+B_{i} \dot{s}_{i}+\mu_{i}(t) K_{i}\left(\beta_{i}-1\right) e_{i}
$$

Where :

$$
\tilde{E}=E-\hat{E}, \quad \bar{A}_{i}=\left[\begin{array}{ccccc}
-\vartheta_{i, 1} & 1 & 0 & \cdots & 0 \\
-\vartheta_{i, 2} \mu_{i}(t) & 0 & 1 & \cdots & 0 \\
\vdots & \vdots & \vdots & \ddots & \vdots \\
-\vartheta_{i, r_{i}-2} \mu_{i}^{r_{i}-3}(t) & 0 & 0 & \cdots & 1 \\
-\vartheta_{i, r_{i}-1} \mu_{i}^{r_{i}-2}(t) & 0 & 0 & \cdots & 0
\end{array}\right]
$$

The matrix $\bar{A}_{i}$ is stable, then it exists $P_{i}=P_{i}^{T}>0$ and $Q_{i}=Q_{i}^{T}>0$, as : [14]

$$
P_{i} A_{i}+A_{i}^{T} P_{i}=-Q_{i} \quad i=\overline{1: p}
$$

\section{1. Proof}

Consider the following Lyapunov function candidate: 


$$
\left\{\begin{array}{l}
L=L_{1}+L_{2} \\
L_{1}=\frac{1}{2} \sum_{i=1}^{P}\left(\tilde{E}_{i}^{T} P_{i} \tilde{E}_{i}\right) \\
L_{2}=\frac{1}{2} \sum_{i=1}^{p}\left(g_{i}^{-1} s_{i}^{2}+\operatorname{tr}\left(\tilde{W}_{i}^{T} F_{W_{i}}^{-1} \tilde{W}_{i}\right)+\operatorname{tr}\left(\tilde{V}_{i}^{T} F_{V_{i}}^{-1} \tilde{V}_{i}\right)+\frac{1}{\gamma_{i}} \tilde{w}_{i}^{2}\right)
\end{array}\right.
$$

After introducing the following equations (13), (18), (19), (25) and (26) and by using the $5^{\text {th }}$ and the $6^{\text {th }}$ assumption the first time derivative of Lyapunov function can express by:

$$
\dot{L} \leq \sum_{i=1}^{p}\left(\begin{array}{l}
-\left.\left(\frac{\mu_{i}(t)}{2} \lambda_{\min }\left(Q_{i}\right)-c_{i, 9}\right)\left|\tilde{E}_{i} \|^{2}-k_{i}\left(1-c_{i, 5}\right)\right| s_{i}\right|^{2} \\
-\left(\frac{\kappa_{i}}{2}-c_{i, 6}\right)\left|\tilde{W}_{i}\left\|^{2}-\left(\frac{\kappa_{i}}{2}-c_{i, 7}\right) \mid \tilde{V}_{i}\right\|^{2}+c_{i, 10}\right.
\end{array}\right)
$$

Where :

$$
\begin{gathered}
c_{i, 4}=\left\|P_{i} B_{i} \Phi_{0 i}\right\|+k_{i}\left\|P_{i} B_{i} g_{i} \Phi_{i}\right\|, \quad c_{i, 5}=\frac{1}{2}\left(\left\|\Phi_{i}\right\|+\frac{k_{i}\left\|P_{i} B_{i} g_{i}\right\|+\beta_{i} \gamma_{i, 1}\left\|P_{i} \mu_{i}(t) K_{i}\right\|}{k_{i}}\right), \\
c_{i, 6}=\frac{c_{i, 1}\left(\left\|\Phi_{i}\right\|+\left\|P_{i} B_{i} g_{i}\right\|\right)}{2}, \quad c_{i, 7}=\frac{c_{i, 2}\left(\left\|\Phi_{i}\right\|+\left\|P_{i} B_{i} g_{i}\right\|\right)}{2}, c_{i, 8}=\frac{\left(c_{i, 3}\left(\left\|\Phi_{i}\right\|+\left\|P_{i} B_{i} g_{i}\right\|\right)+\beta_{i} \gamma_{i, 2}\left\|P_{i} \mu_{i}(t) K_{i}\right\|\right)}{2}, \\
c_{i, 9}=c_{i, 4}+k_{i} c_{i, 5}+c_{i, 6}+c_{i, 7}+1, \quad c_{i, 10}=c_{i, 8}^{2}++\frac{\kappa_{i}}{2} \mid \hat{s}_{i}\left(\left\|W_{i}\right\|^{2}+\left\|V_{i}\right\|^{2}\right) .
\end{gathered}
$$

Let's suppose that: $c_{i, 5}<1, \quad{ }_{i}(t)>c_{i, 9} / \lambda_{\min }\left(Q_{i}\right)$ and $\kappa_{i}>2 \operatorname{Max}\left(c_{i, 6}, c_{i, 7}\right)$. So the Lyapunov function is decreasing if $s_{i}, \widetilde{E}_{i}, \quad \tilde{W}_{i}$ and $\tilde{V}_{i}$ are outsiders of the compact sets $\Omega_{s_{i}}, \Omega_{\tilde{E}_{i}}, \Omega_{\tilde{W}_{i}}$ and $\Omega_{\tilde{V}_{i}}$ respectively, where:

$$
\left\{\begin{array}{l}
\Omega_{s_{i}}=\left\{s_{i} /\left|s_{i}\right| \leq \sqrt{\left.\frac{c_{i, 10}}{k_{i}\left(1-c_{i, 5}\right)}\right\},}\right. \\
\Omega_{\tilde{E}_{i}}=\left\{\tilde{E}_{i} /\left\|\tilde{E}_{i}\right\| \leq \sqrt{\frac{2 c_{i, 10}}{\mu_{i}(t) \lambda_{\min }\left(Q_{i}\right)-2 c_{i, 9}}}\right\} \\
\Omega_{\tilde{W}_{i}}=\left\{\tilde{W}_{i} /\left\|\tilde{W}_{i}\right\| \leq \sqrt{\frac{2 c_{i, 10}}{\kappa_{i}-2 c_{i, 6}}}\right\}, \\
\Omega_{\tilde{V}_{i}}=\left\{\tilde{V}_{i} /\left\|\tilde{V}_{i}\right\| \leq \sqrt{\frac{2 c_{i, 10}}{\kappa_{i}-2 c_{i, 7}}}\right\}
\end{array}\right.
$$

\section{Simulation RESUltS}

The simulation experiments are performed to compare the ordinary high gain observe and the proposed observer.

The simulation parameters are given in Tables I and II. The $3^{\text {rd }}$ Figure show simulation results obtained by using the proposed and the conventional approach at the presence of noise 
measurement and with 50\% parametric variation from time $t=10 \mathrm{sec}$.

Table 1: Quadrotor Parameters.

\begin{tabular}{cccc}
\hline Symbol & Value & Symbol & Value \\
\hline$m$ & $0.486(\mathrm{Kg})$ & $I_{z}$ & $7.65 \mathrm{e}-3\left(\mathrm{KgM}^{2}\right)$ \\
$l$ & $0.25(\mathrm{~m})$ & $g_{f t}$ & $9.81\left(\mathrm{Ms}^{2}\right)$ \\
$k_{d}$ & $3.23 \mathrm{e}-7\left(\mathrm{NMs}^{2}\right)$ & $k_{f t x}, k_{f t y}$ & $5.56 \mathrm{e}-4(\mathrm{Ns} / \mathrm{M})$ \\
$k_{p}$ & $2.98 \mathrm{e}-5\left(\mathrm{Ns}^{2}\right)$ & $k_{f t z}$ & $6.35 \mathrm{e}-4(\mathrm{Ns} / \mathrm{M})$ \\
$J r$ & $2.83 \mathrm{e}-5\left(\mathrm{KgM}^{2}\right)$ & $k_{f r \varphi}, k_{f r \theta}$ & $5.56 \mathrm{e}-4(\mathrm{Ns} / \mathrm{r})$ \\
$I_{x}$ & $3.82 \mathrm{e}-3\left(\mathrm{KgM}^{2}\right)$ & $k_{f r u}$ & $6.35 \mathrm{e}-4(\mathrm{Ns} / \mathrm{r})$ \\
$I_{y}$ & $3.82 \mathrm{e}-3\left(\mathrm{KgM}^{2}\right)$ & & \\
\hline
\end{tabular}

Table 2: Controller and Observer Parameters.

\begin{tabular}{|c|c|c|c|}
\hline \multicolumn{2}{|c|}{ Controller } & \multicolumn{2}{|c|}{ Proposed Observer } \\
\hline Symbol & Value & Symbol & Value \\
\hline $\begin{array}{c}\lambda_{\eta} \\
\lambda_{\xi} \\
K_{\xi} \\
K_{\eta} \\
\lambda_{x}, \lambda_{y}, \\
\lambda_{z} \\
\lambda_{\varphi}, \lambda_{\theta} \\
, \lambda_{\psi} \\
F_{W x, y, z} \\
F_{W \theta, \varphi, \psi} \\
F_{V x, y, z} \\
F_{V \theta, \varphi, \psi} \\
\kappa_{x}, \kappa_{y}, \\
\kappa_{z} \\
\kappa_{\varphi}, \kappa_{\theta} \\
, \kappa_{\psi}\end{array}$ & $\begin{array}{c}\operatorname{daig}(10,10,10) \\
\operatorname{diag}(1,1,10) \\
\text { daig }(1.5,1.5,20) \\
\operatorname{diag}(0.2,0.2,0.2) \\
0.01 \\
0.01 \\
0.01 \\
0.01 \\
3 I_{3.3} \\
I_{3.3} \\
5 I_{5.5} \\
I_{5.5} \\
0.1 \\
0.1 \\
0.1 \\
0.1\end{array}$ & $\begin{array}{c}x, y, z, f \\
\theta, \varphi, \varphi, f \\
x, y, z, 1 \\
\theta, \varphi, \varphi, 1 \\
x, y, 2 \\
\theta, \varphi, z, 2 \\
\psi,, 2 \\
\delta_{x, y, \varphi, \theta} \\
\delta_{z} \\
\delta_{\psi} \\
\vartheta_{x, y, z, 1} \\
\vartheta_{x, y, z, 2} \\
\vartheta_{\theta, \varphi, \psi, 1} \\
\vartheta_{\theta, \varphi, 2} \\
\vartheta_{\psi, 2} \\
\beta_{x, y, z} \\
\beta_{\theta, \varphi, \varphi} \\
\bar{\varepsilon}_{x, y, z, \varphi, \theta} \\
\bar{\varepsilon}_{\psi}\end{array}$ & $\begin{array}{c}2 \\
2 \\
10 \\
5 \\
0.1 \\
0.2 \\
0.3 \\
20 \\
30 \\
40 \\
10 \\
40 \\
5 \\
20 \\
30 \\
0.5 \\
0.8 \\
0.01 \\
0.05\end{array}$ \\
\hline
\end{tabular}

The conventional high gain observer produces large measurement noise amplification has created degradations in system's pursuit and by consequence an important control signal as is presented as in Figure.3. a which can cause instability if the measurement noise is so important.

Unlike the results achieved by the proposed observer where a significant minimization of unfavorable effect was obtained for the system output and control signals as is shown in the Figure. 3. b where control signal amplitude is ten times less than the one gotten by using ordinary observer. 
International Journal of Information Technology, Control and Automation (IJITCA) Vol.2, No.3, July 2012

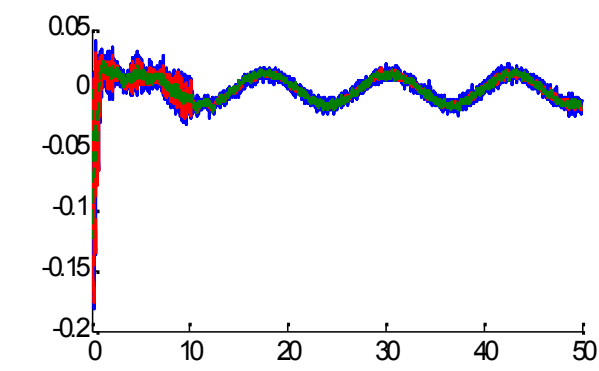

(3. a. 1)

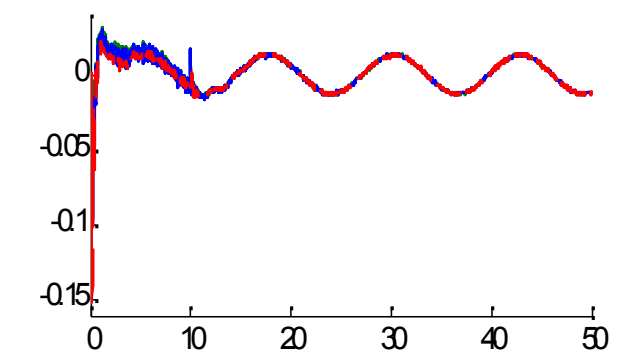

(3. b. 1)

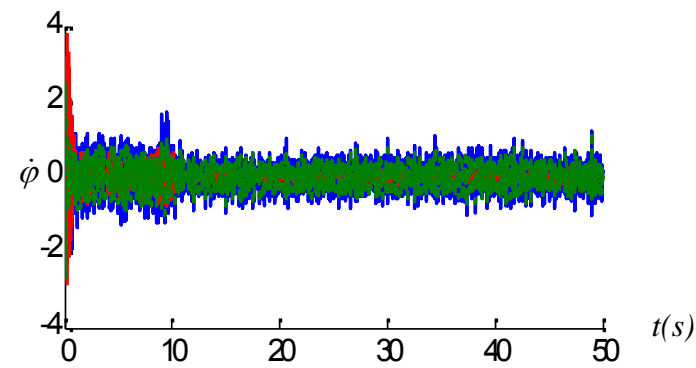

(3. a. 2)

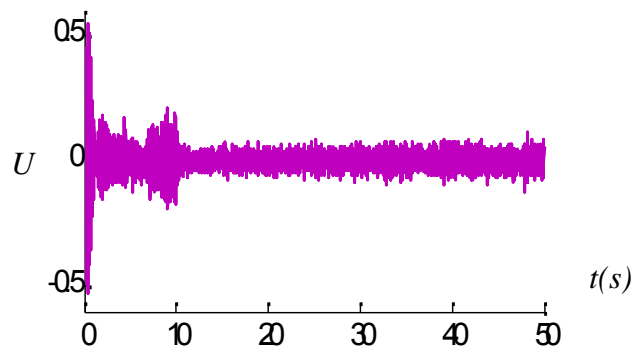

(3. a. 3)

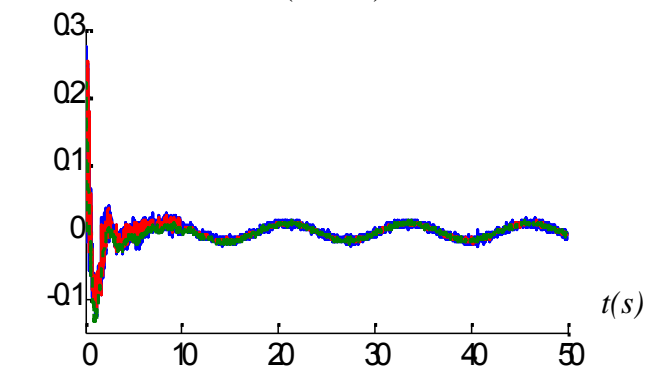

(3. a. 4)

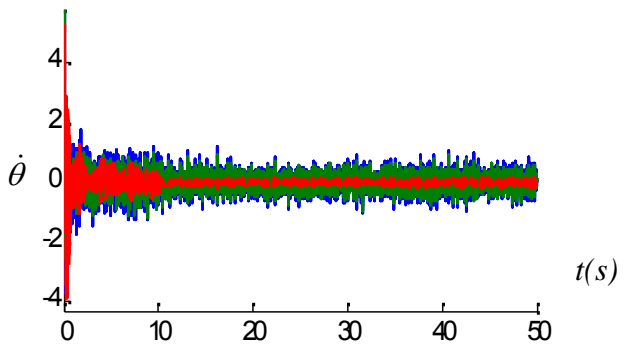

(3. a. 5)

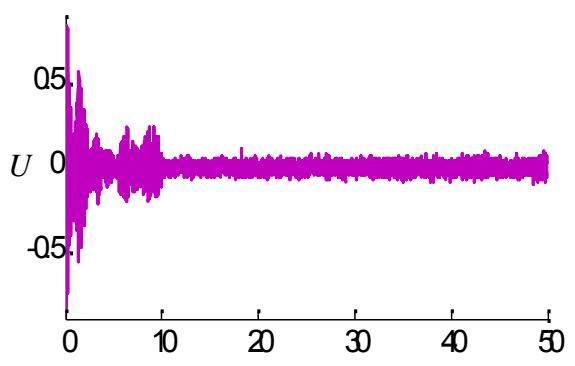

$t(s)$

(3. a. 6)

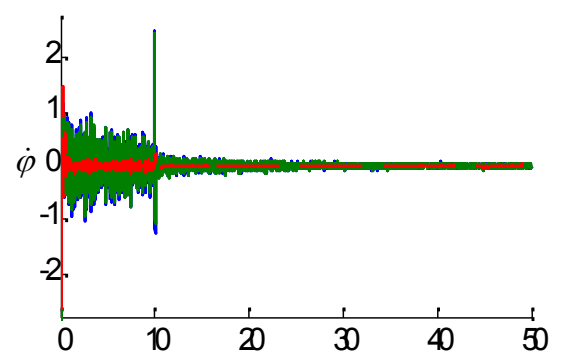

$t(s)$

(3. b. 2)

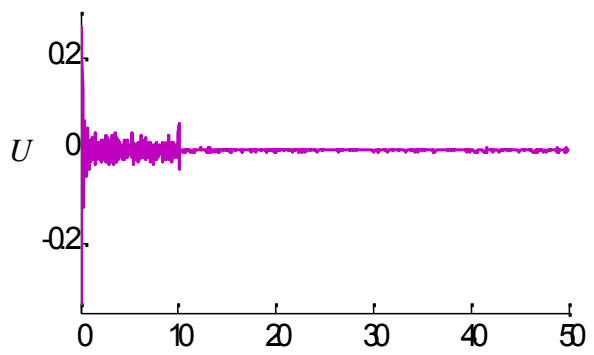

$t(s)$

(3. b. 3)

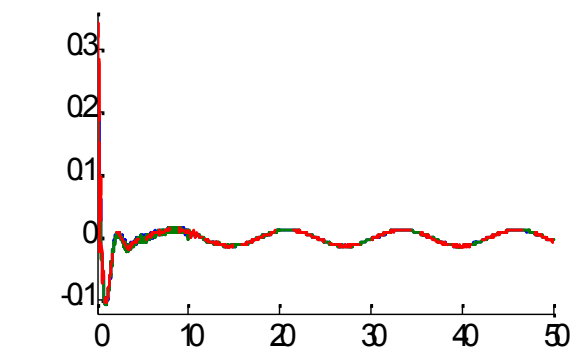

$t(s)$

(3. b. 4) 


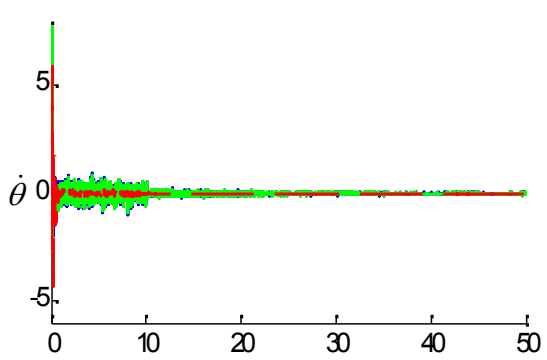

(3. b. 5)

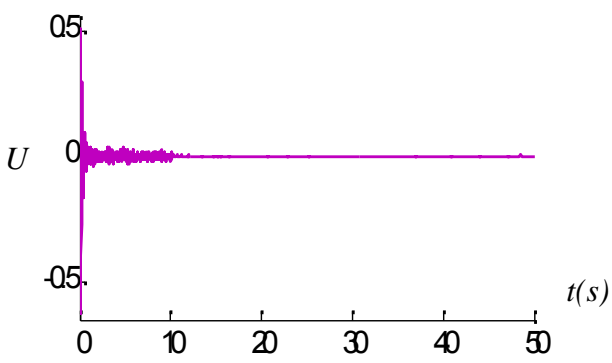

(3. b. 6)

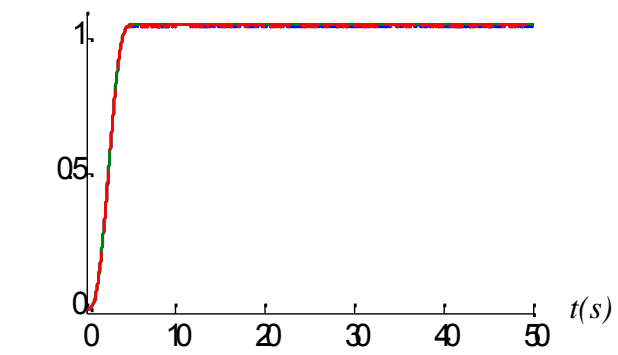

(3. a. 7)

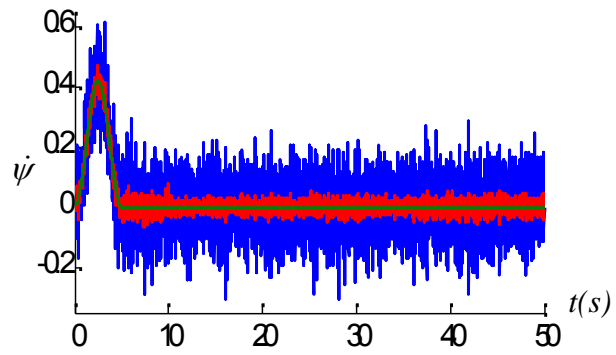

(3. a. 8)

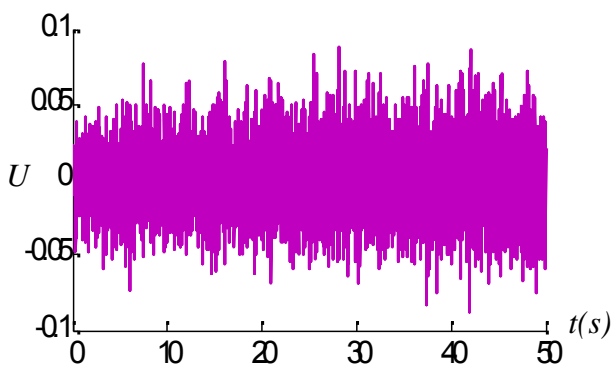

(3. a. 9)

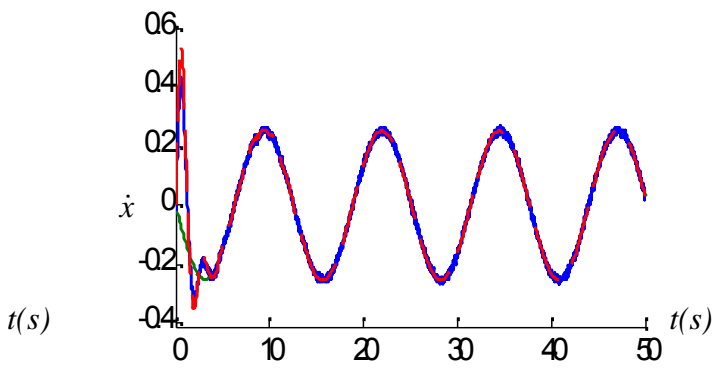

(3. a. 10)

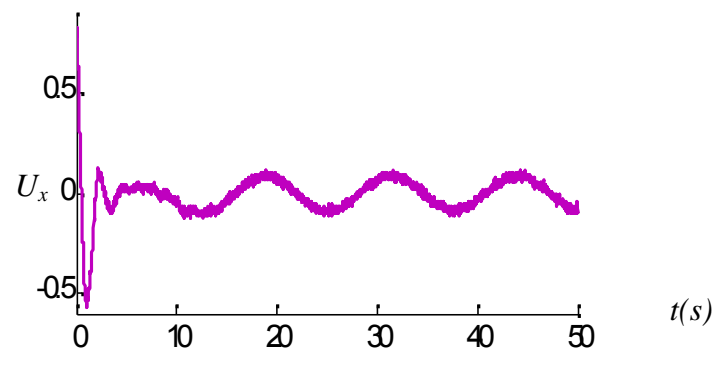

(3. a. 11)

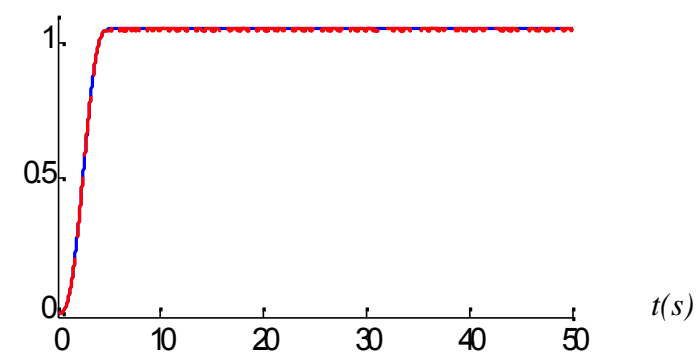

(3. b. 7)

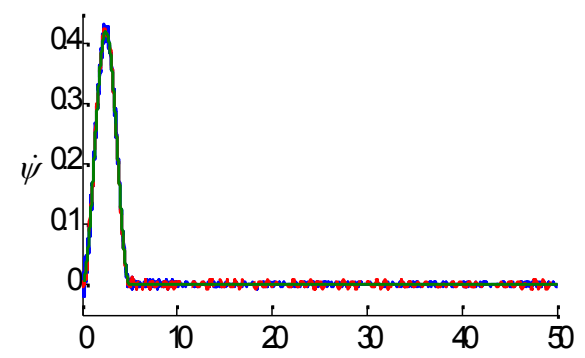

$t(s)$

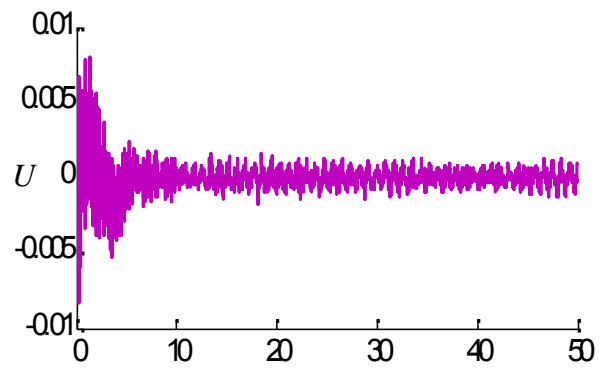

$t(s)$ 
International Journal of Information Technology, Control and Automation (IJITCA) Vol.2, No.3, July 2012

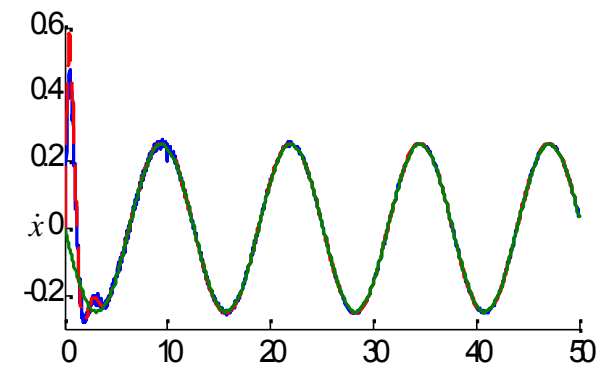

(3. b. 10)

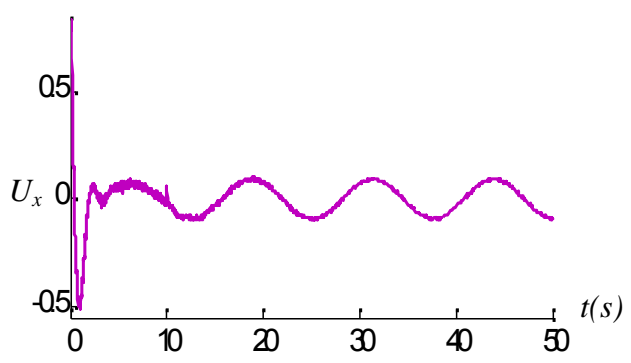

(3. b. 11)

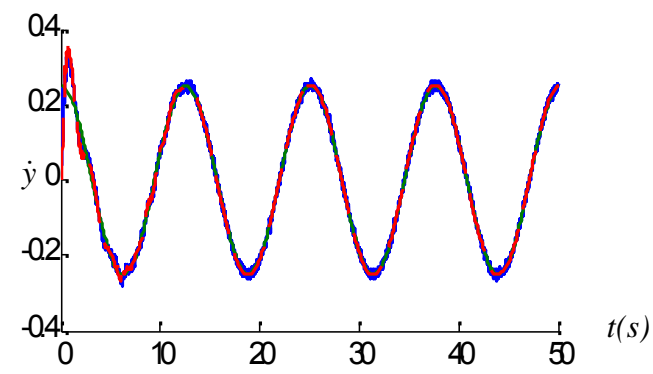

(3. a. 12)

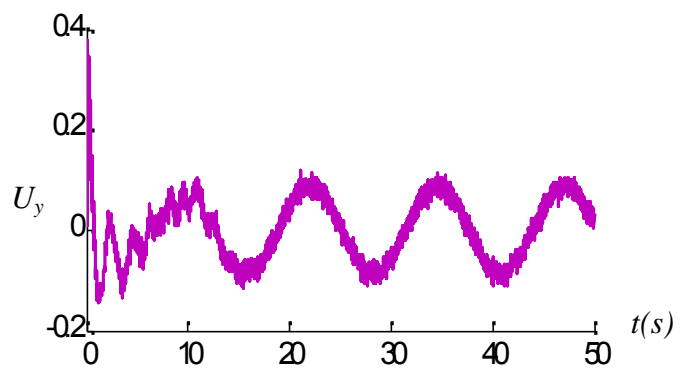

(3. a. 13)

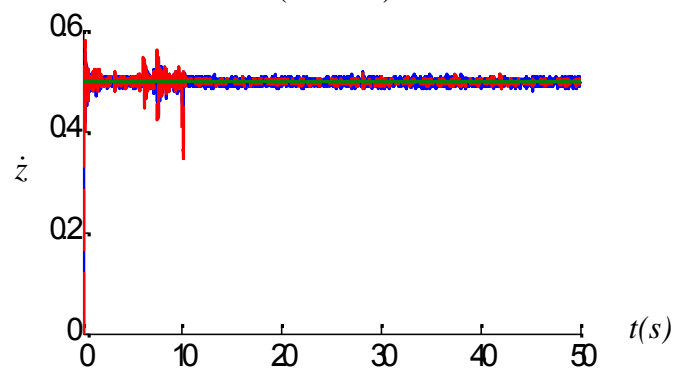

(3. a. 14)

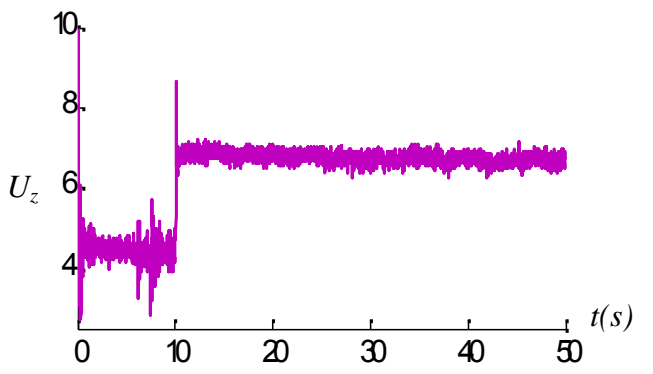

(3. a. 15)

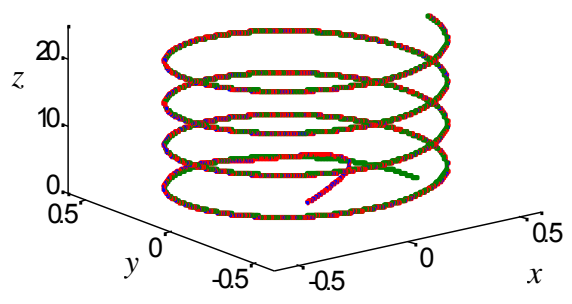

(3. a. 16)

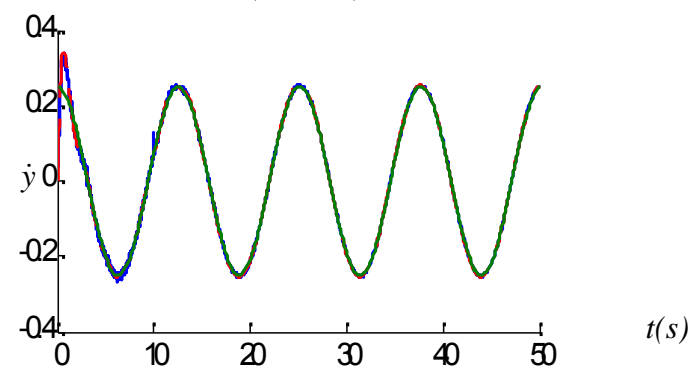

(3. b. 12)

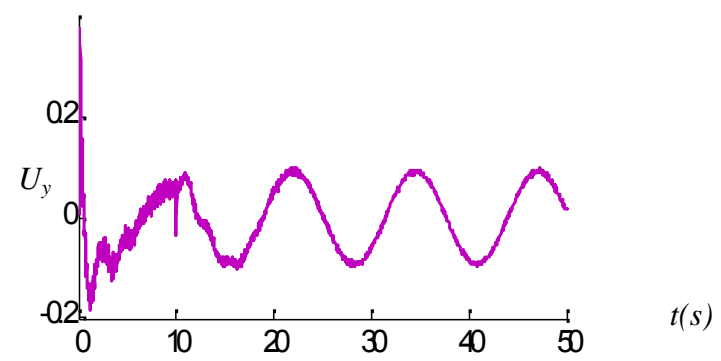

(3. b. 13)

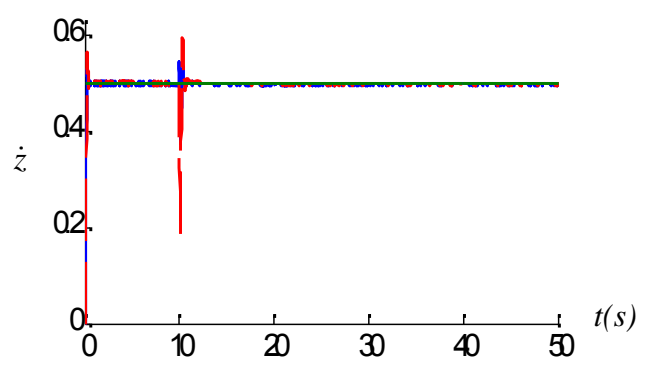

(3. b. 14) 


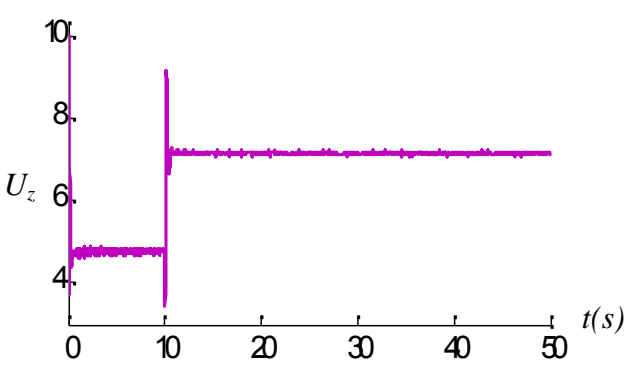

(3. b. 15)

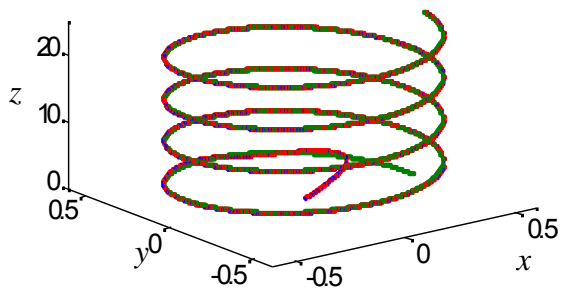

(3. b. 16)

Figure. 3: Simulation results

\section{CONCLUSION}

A new adaptive observer was developed for an under actuated Quadrotor UAV. The control scheme is based on the use of SHL neural networks for each Quadrotor subsystem in order to estimate on line the unknown nonlinear dynamical modal in Quadrotor helicopter. The proposed observer is based on conventional high gain observer structure where new parameters are added to the last one in order to reduce the amplification of measurement noise generated by ordinary observer. The proposed observer have proved its capacity where a very important minimization in the amplitude and the variance of the noisy signal is gotten without having degradations in the performances as it was shown by the numeric simulation results. This method does not require any prior knowledge about dynamic model and states variables. The uniformly ultimately boundedness of the tracking error and all signals in the overall closed-loop system is proved using Lyapunov's direct method. The effectiveness of the proposed approach was validated by simulation of a Quadrotor control at noise measurement presence.

\section{REFERENCES}

[1] P. Adigbli : Nonlinear Attitude and Position Control of a Micro Quadrotor using Sliding Mode and Backstepping Techniques. 3rd US-European Competition, MAV07 \& EMAV07, 17-21, France, 2007

[2] S.Bouabdallah and al : Backstepping and sliding mode Techniques Applied to an Indoor Micro Quadrotor. In Proc 2005 IEEE ICRA, 2005

[3] T. Madani and A.Benallegue: Backstepping Control with Exact 2-Sliding Mode Estimation for a Quadrotor Unmanned Aerial Vehicle. Proc of IEEE/RSJ International CIRS, 2007.

[4] A.Mokhtari, A.Benallegue and B.Daachi: Robust Feedback Linearization and GH $\infty$ Controller for a Quadrotor Unmanned aerial vehicle. Journal of Electrical Engineering, vol.57, No.1, pp 20.27, 2006.

[5] S.Bouaddallah and al : PID vs. LQ control Techniques Applied to an Indoor Micro Quadrotor. In Proc IEEE ICIRS, Japan, 2004.

[6] A.Das, F.Lewis and K.Subbarao : Backstepping Approach for Controlling a Quadrotor Using Lagrange Form Dynamics. J Intell Robot syst, pp 127-151, 2009.

[7] C. Nicol, \& al: Robust Neural Network of a Quadrotor Helicopter, in proc IEEE Fuzzy information PSC, pp. 454-458, 2008.

[8] J.C.Raimundez \& al : Adaptive Tracking control for a Quad-rotor. ENOC-2008, saint Pertersburg, Russia July 2008.

[9] O.Bouhali and H.Boudjedir : Neural Network control with neuro-sliding mode observer applied to Quadrotor Helicopter. In Proc INISTA’11, pp 24-28, Istanbul, Turkey 2011.

[10] T.Dieks and S.Jagannathan: Neural Networks Out Feedback of a Quadrotor UAV. Proc IEEE, 47th CDC, pp 3633-3639, Mexi, Dec 2008.

[11] H.Khalil : High-Gain Observers in Nonlinear Feedback Control. Proc IEEE, IC on Control, Automation and Systems, 2008 
[12] M.Farza and al : Dynamic High Gain Observer Design. Proc IEEE, 6th International MultiConference on systems, signals and Device, 2009.

[13] E.Bullinger and F.Allgower : An Adaptive High-Gain Observer for Nonlinear Systems. Proc IEEE, the 36th CDC USA, 1997.

[14] J.J.Slotine : Applied nonlinear control. Prentice Hall, 1991.

[15] S.Jagannathan : Neural networks control of nonlinear Discrete-time systems. CRC press, Taylor \& Francic Group, 2006. 